\title{
Folgeerkrankungen durch konsequente Therapie verhindern
}

- Typischerweise beginnt die Symptomatik der Gicht am Großzehengrundgelenk, kann aber auch andere Gelenke betreffen. „Bei Patienten mit ungeklärten Gelenkbeschwerden ist die Gicht aufgrund ihrer Häufigkeit immer eine Differenzialdiagnose, die es zu beachten gilt", berichtete Prof. Ulf Müller-Ladner, Bad Nauheim. Unbehandelt hat die Gicht einen chronisch-progredienten Verlauf. Früher Beginn, lange Krankheitsdauer, inadäquate Therapie, erhöhte Serum-Harnsäurewerte, hohe Anfallsfrequenz und polyartikulärer Befall sind Risikofaktoren für die Entwicklung einer tophösen Gicht.

Die Gichtmanifestationen bleiben aber nicht auf Gelenke beschränkt, hob Dr. AnneKathrin Tausche, Dresden, hervor. Etwa einer von 1000 Gichtpatienten entwickelt extrazelluläre Manifestationen der tophösen
Gicht. So wurden Harnsäureablagerungen im Auge nachgewiesen. Sie waren mit einer Visusverminderung assoziiert und bildeten sich unter einer suffizienten harnsäuresenkenden Therapie zurück. Auch im Larynx, in der Lunge und im Herzen (v. a. an der Mitralklappe) hat man Gichttophi gefunden. In der Regel handelte es sich dabei um Zufallsbefunde. In extrem seltenen Fällen wurden auch viszerale Tophi beschrieben.

\section{Bei suspekter Klinik: an seltene} Gichtmanifestation denken!

Ähnlich wie die Gelenkgicht bilden sich auch die extraartikulären Gichtmanifestationen unter einer adäquaten, konsequenten Therapie zurück. Wichtig sei, so Tausche, dass man bei entsprechend suspekter Klinik an die Möglichkeit einer seltenen Gichtmanifestation denke und diese weiter abkläre. Zudem können erhöhte Harnsäurewerten zur Entwicklung von Nierensteinen führen und den Blutdruck beeinflussen, berichtete PD Dr. Jan T. Kielstein, Hannover.

Langzeitstudiendaten zeigen, dass die Serumharnsäure unter Febuxostat (Adenuric $^{\oplus}$ ) über mindestens fünf Jahre unter dem empfohlenen Zielwert von $6,0 \mathrm{mg} / \mathrm{dl}$ liegt (Schumacher HR et al. Rheumatology 2009; 48: 188-194). Unter kontinuierlicher Therapie mit dem selektiven XanthinoxidaseHemmer erlitten die Patienten nahezu keine neuen Anfälle. Die Tophi wurden deutlich kleiner und bildeten sich bei $79 \%$ der Patienten sogar komplett zurück.

- Abdol A. Ameri

Quelle: Fachpressegespräch „Arthritis urica und Folgeerkrankungen", Berlin, Juni 2011 (Veranstalter: Berlin-Chemie)

\section{Zulassungerweiterung für Dalteparin Rezidivprophylaxe tiefer Venenthrombosen auch bei onkologischen Patienten}

- Durch das deutlich erhöhte Rezidivrisiko für venöse Thromboembolien (VTE) bei onkologischen Patienten ist die Sekundärprophylaxe in dieser Indikation von hoher praktischer Relevanz. Dalteparin erhielt als erstes niedermolekulares Heparin (NMH) in Deutschland die Zulassungserweiterung zur VTE-Rezidivprophylaxe bei diesen Patienten.

Die Ergebnisse der CLOT-Studie zeigten, dass die Rezidivrate mit dem NMH Dalteparin $\left(\right.$ Fragmin $\left.^{\oplus}\right)$ im Vergleich zu oralen Antikoagulanzien (Vitamin-K-Antagonisten) um die Hälfte verringert werden kann ( $p=$ 0,002) (Lee AY, et al. N Engl. J Med 2003; 349: 146-53). An der prospektiven, offenen und randomisierten Studie nahmen 676 Patienten über sechs Monate teil. Von diesen Patienten wiesen $90 \%$ solide Tumoren und $67 \%$ Metastasen auf (ECOG-Status 1-2). Bei allen Teilnehmern wurde vor Aufnahme in die Studie eine akute Thrombo- embolie diagnostiziert. Die Gesamtblutungsraten waren mit 13,6\% (Dalteparin) und $18, \%$ (Cumarin) vergleichbar. Auch schwere Blutungen waren mit 5,6\% bzw. $3,6 \%$ nicht signifikant verschieden.

Vitamin-K-Antagonisten (VKA) sind für onkologische Patienten weniger gut geeignet, da Wechselwirkungen (z. B. mit Zy-

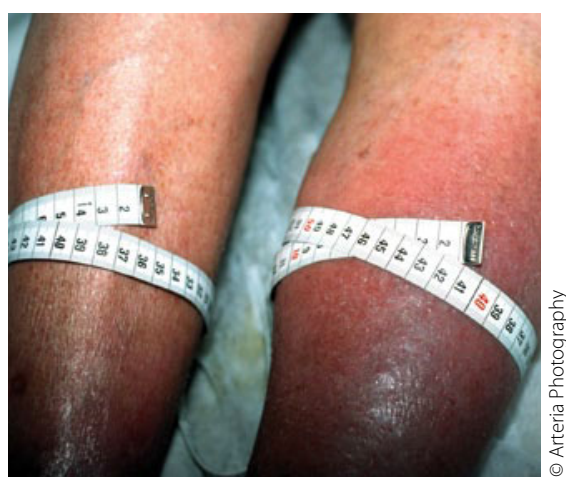

Umfangsmessung bei Beinvenenthrombose links. tostatika), eine veränderte Leberfunktion sowie gastrointestinale Störungen (Erbrechen, Durchfall) die Wirkung der oral aufgenommenen Substanzen beeinträchtigen können. Aufgrund der verzögerten und anhaltenden gerinnungshemmenden Wirkung der VKA kann außerdem die Anwendung für Patienten, die sich regelmäBig invasiven Verfahren (z. B. Lumbalpunktionen) unterziehen müssen oder häufiger eine chemotherapieinduzierte Thrombozytopenie entwickeln, schwierig sein.

In den aktuellen S2-Leitlinien zur Diagnostik und Therapie der tiefen Venenthrombose und Lungenembolie findet sich dementsprechend eine Empfehlung zur Behandlung von Thrombosen bei Tumorpatienten, die anstelle von Vitamin-K-Antagonisten für drei bis sechs Monate mit $\mathrm{NMH}$ behandelt werden sollten.

- Red.

Quelle: Nach Informationen von Pfizer 